\title{
Ultrasonographic Evaluation of the Kidney in 5/6 Nephrectomized Rats: Correlation with Biochemical and Histopathological Findings
}

\author{
ANTÓNIO NOGUEIRA ${ }^{1,2}$, ANA FAUSTINO ROCHA ${ }^{1,3}$, MARIO GINJA $^{1,3}$, \\ PAULA ALEXANDRA OLIVEIRA ${ }^{1,3}$ and MARIA JOÃO PIRES ${ }^{1,3}$ \\ ${ }^{1}$ Department of Veterinary Sciences, School of Agrarian and Veterinary Sciences, and \\ ${ }^{3}$ Center for the Research and Technology of Agro-Environmental and Biological Science, \\ University of Trás-os-Montes and Alto Douro, Vila Real, Portugal; \\ ${ }^{2}$ Department of Therapeutic and Diagnostic Technologies, Polytechnic Institute of Bragança, Bragança, Portugal
}

\begin{abstract}
Aim: To characterize the kidney alterations associated with renal mass reduction by two-dimensional ultrasound and assess the correlation of the ultrasonographic parameters with the histological and biochemical findings. Materials and Methods: Rats were divided into two groups: sham-operated animals $(n=13)$, and animals which underwent renal mass reduction (RMR) through 5/6 nephrectomy (n=14). Renal length, renal thickness, cortical thickness, medullary length and echogenicity of the kidneys were evaluated by ultrasonography at 3 and 6 months after the RMR. Results: Except for the renal length, the renal dimensions at 3 and 6 months were significantly higher in the RMR group when compared to the sham-operated group $(p<0.05)$. Furthermore, the cortical and medullary echogenicity was significantly higher in the RMR group when compared to the sham-operated group $(p<0.05)$. A significant correlation was observed between the plasma creatinine level and the renal length 3 months after RMR ( $r=-0.612$, $p=0.045)$. Conclusion: These data support future application of ultrasonography for monitoring the progression of renal damage in chronic studies with the 5/6 nephrectomy model.
\end{abstract}

Chronic kidney disease (CKD) is a major public health problem affecting approximately $8-16 \%$ of the population worldwide (1). Animal models are important tools in studying CKD. The renal mass reduction (RMR) animal model has

Correspondence to: Maria João Miranda Pires, Department of Veterinary Sciences, University of Trás-os-Montes and Alto Douro, 5001-801 Vila Real, Portugal. Tel: +351 259350655, Mob: +351 965241536,e-mail: joaomp@utad.pt

Key Words: Wistar rats, 5/6 nephrectomy model, ultrasonography, kidney size, echogenicity. been a mainstay of studies of progressive CKD, mainly in studying the pathogenesis and novel therapy of kidney diseases (2, 3). After 5/6 nephrectomy, animals develop several kidney alterations, such as glomerular hypertrophy, tubular atrophy, glomerular and interstitial fibrosis, and renal insufficiency (4-7).

In the model of RMR, assessing renal dysfunction and histopathological alterations is of great importance in the evaluation of new therapeutic approaches for $\operatorname{CKD}(8,9)$. In humans, renal biopsy has been advocated in patients with CKD $(10,11)$. However, its invasive nature and the need for serial biopsies in order to monitor progression of the disease would expose animal models to repeated risks and is not practical. Therefore, certain biochemical markers, such as blood urea nitrogen (BUN), creatinine, proteinuria and creatinine clearance, are typically used to assess renal dysfunction in this model of CKD $(8,9)$. However, laboratory detections primarily demonstrate functional abnormalities of the kidneys.

Two-dimensional ultrasonography (US), an established non-invasive imaging technique, is appealing because of its simplicity, low cost, non-invasiveness and safety, widespread availability and non-nephrotoxicity (12-15). Studies have demonstrated that transabdominal renal US is both repeatable and reproducible $(16,17)$ and it is routinely performed in animals with suspected renal disease (18). The distinction between normal kidney and kidney with chronic damage by two-dimensional US imaging is currently based on morphological changes, including in size and parenchymal echogenicity. Increased cortical or cortical and medullary echogenicity is reported to be one of the most common signs of chronic or acute kidney disease in veterinary medicine (18). In human medicine, a high correlation between cortical echogenicity and glomerular sclerosis, tubular atrophy, interstitial fibrosis and interstitial inflammation has been 
described (19). Consequently, US may be a potential tool for monitoring the progression of renal damage in the RMR model.

The aims of the current study were to characterize kidney alterations associated with RMR in Wistar rats by twodimensional US, and compare renal dimensions obtained by this method with post-mortem measurements. We also assessed the correlation of the US parameters with the histological and biochemical changes during the progression of kidney disease in this model.

\section{Materials and Methods}

Animals. Thirty male Wistar rats (Rattus norvegicus) of 4 weeks of age (112-157 g body weight) were acquired from Harlan Interfauna Inc. (Barcelona, Spain). Animals were housed in filter-capped polycarbonate cages (Tecniplast, Buguggiate, Italy) with corncob for bedding (Mucedola, Milan, Italy) at the University of Trás-osMontes and Alto Douro. All cages were maintained on a 12/12-h light-dark cycle in a ventilated room under controlled conditions of temperature $\left(23 \pm 2^{\circ} \mathrm{C}\right)$ and relative humidity $(50 \pm 10 \%)$. Cages were cleaned once per week and water was changed weekly. Animals had ad libitum access to a basic standard diet (4RF2 $1{ }^{\circledR}$; Mucedola) and bottled water throughout the study.

Surgical procedure and experimental groups. After a period of acclimation of 7 weeks, each rat was weighed (359 to $402 \mathrm{~g}$ ) and anesthetized by intraperitoneal administration of ketamine (Imalgene ${ }^{\circledR} 1000,70 \mathrm{mg} / \mathrm{kg}$; Merial S.A.S., Lyon, France) and xylazine (Rompum ${ }^{\circledR} 2 \%, 10 \mathrm{mg} / \mathrm{kg}$; Bayer S.A., Kiel, Germany). RMR was performed in 17 rats. The animals were subjected to right nephrectomy and surgical removal of the left kidney poles (8). A sham operation was performed on 13 additional rats as a nonnephrectomized control (sham-operated; SO). One week after surgery, surviving animals were randomly divided into SO $(n=13)$ and RMR ( $n=14)$ groups. All procedures followed the European (European Directive 2010/63/EU) and National (Decree-Law $113 / 2013$ ) legislation on the protection of the animals used for scientific purposes.

Ultrasonographic evaluation. At 3 and 6 months after the surgical procedure, the left kidney of each animal was evaluated by ultrasonography using two-dimensional US (B mode). For this, the left flank of each animal was shaved using a machine clipper (AESCULAP ${ }^{\circledR}$ GT420 Isis; Aesculap Inc., Center Valley, PA, USA). Animals were restrained in prone position without anesthesia and sagittal US images of the kidney were obtained under the same conditions in all animals using acoustic gel (Aquasonic ${ }^{\circledR}$; Parker Laboratories Inc., NJ, USA) and a real time scanner (LogiqP6 ${ }^{\circledR}$; General Electric Healthcare, WI, USA) with a $10 \mathrm{MHz}$ linear probe. The images were recorded for subsequent analysis. For each image, the renal length was measured as the greatest pole to pole distance and the renal thickness was measured as the distance between the renal capsule and pelvis, perpendicular to the renal length. The cortical thickness was measured as the distance from the renal capsule to the base of medullar pyramid. Additionally, the medullary length was measured perpendicular to the medullary thickness (Figure 1). The mean cortical and medullary echogenicity was evaluated; for this, an area of approximately $1 \mathrm{~cm}^{2}$ was evaluated in cortex and medulla using the standard histogram function of Adobe Photoshop CC (Adobe systems Inc., San Jose, CA, USA) (Figure 2).

Sample collection. Blood and urine samples were collected at 3 and 6 months. For urine sample collection, animals were placed in metabolic cages with free access to food and water for 2 days in order to accustom them to isolation conditions. Twenty-four-hour urine samples were collected during the next 2 days to determine urine volume. Subsequently, urine samples were centrifuged at 1500 $\times g$ for $15 \mathrm{~min}$ and stored at $-20^{\circ} \mathrm{C}$ for determination of creatinine and protein concentration. Blood samples $(150 \mu \mathrm{l}$ per animal $)$ were obtained from a cut in the tail tip directly into heparinized capillary tubes. The tubes were centrifuged at $1,500 \times g$ for $15 \mathrm{~min}$, plasma was stored at $-20^{\circ} \mathrm{C}$ to determine creatinine concentration. Glomerular filtration rate was estimated from the creatinine clearance, which was calculated by employing a standard formula (urinary creatinine $\times$ urinary volume/serum creatinine).

Animal sacrifice. Six months after surgery, all animals were anesthetized with isoflurane and humanely sacrificed. A complete necropsy was performed; the remnant kidney from RMR rats or both kidneys from rats of the SO group were removed, measured with a ruler after sagittal sectioning (anatomopathological measurement) and immersed in 10\% buffered formalin for $24 \mathrm{~h}$.

Histopathology. After fixation, the kidneys from all animals were cut and embedded in paraffin blocks. Subsequently, 2- $\mu$ m sections were obtained and mounted on glass slides. For morphometric evaluation, sections were stained with hematoxylin and eosin (H\&E), Reticulin and Masson's trichrome and evaluated blindly by two pathologists under a light microscope. Renal fibrosis was evaluated and scored as reported previously by Asaba et al. (20).

Statistical analysis. A descriptive analysis was performed for all variables included in the study. Data were statistically analyzed with SPSS $^{\circledR}$ (version 23 for Windows; IBM Corp., Armonk, NY, USA) using the independent and paired $t$-test. Spearman correlation was used to assess the correlation between US, histological and biochemical data. Data are presented as the mean \pm standard deviation (S.D.). A $p$-value lower than 0.05 was considered statistically significant.

\section{Results}

Animals. Two animals from the RMR group died (9 and 18 weeks, respectively) after surgery. The data from these animals were not included in the study and the size of the group was reduced to 12 animals in the RMR group. Due to the fact that the US examination was performed in awake animals, adequate images of the kidney could not be obtained in one animal from each experimental group. In this way, only data from 12 animals of the SO group and 11 animals of the RMR group are presented.

Renal measurements by US at 3 and 6 months of the protocol. Statistically significant differences were observed between SO and RMR groups for renal thickness and 

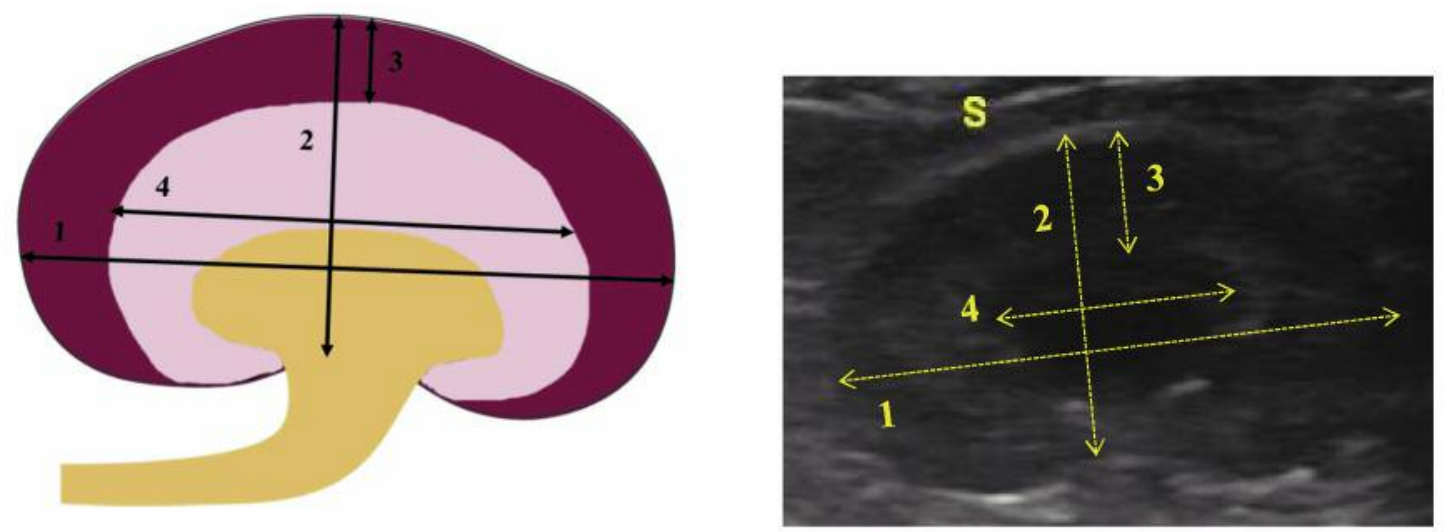

Figure 1. Schematic (A) and ultrasonographic (B) imaging of a normal rat kidney (sham-operated group) in sagittal section (S) (1: renal length; 2: renal thickness; 3: renal cortical thickness; 4: renal medullary length).
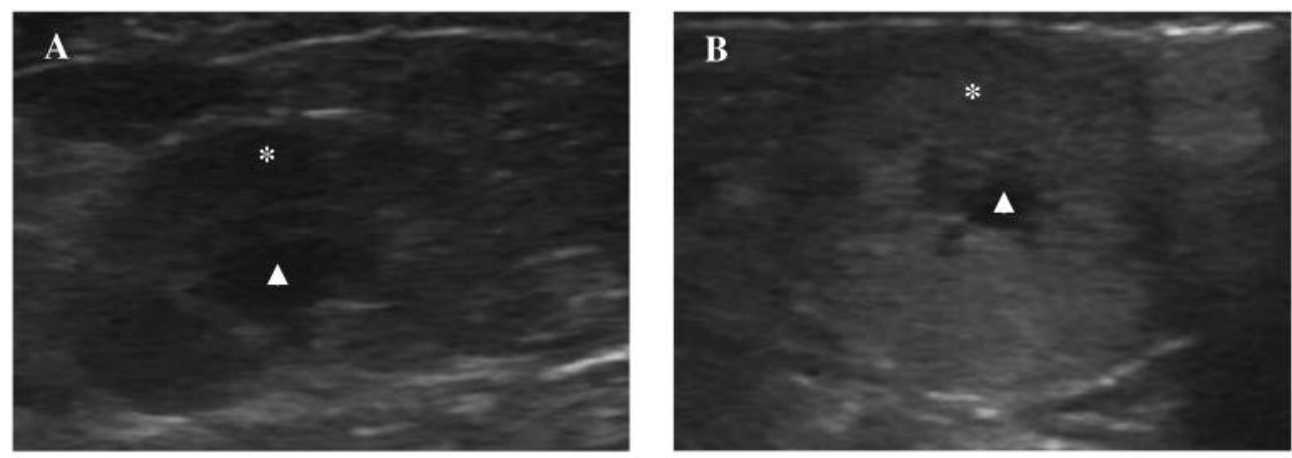

Figure 2. Ultrasonographic images of the kidney in sham-operated (A) and renal mass reduction (B) groups 6 months after 5/6 nephrectomy, revealing the renal cortex (asterisk) and renal medulla (arrowhead).

medullary length at 3 months of the protocol, and for renal thickness, cortical thickness and medullary length at 6 months of the protocol $(p<0.05)$ (Table I). For each experimental group, the renal measurements were higher at 6 months when compared with those at 3 months of the protocol. However, statistically significant differences were only found for renal length in both SO and RMR groups, and for cortical thickness in the RMR group $(p<0.05)$ (Table I).

Comparison between renal measurements using a ruler and by US at 6 months of the protocol. The renal measurements using a ruler were lower when compared to renal measurements obtained by US (Table II). Statistically significant differences were found between US and anatomopathological measurements of renal length in the RMR group $(p<0.05)$ and of renal thickness in both SO and RMR groups $(p<0.05)$ (Table II).

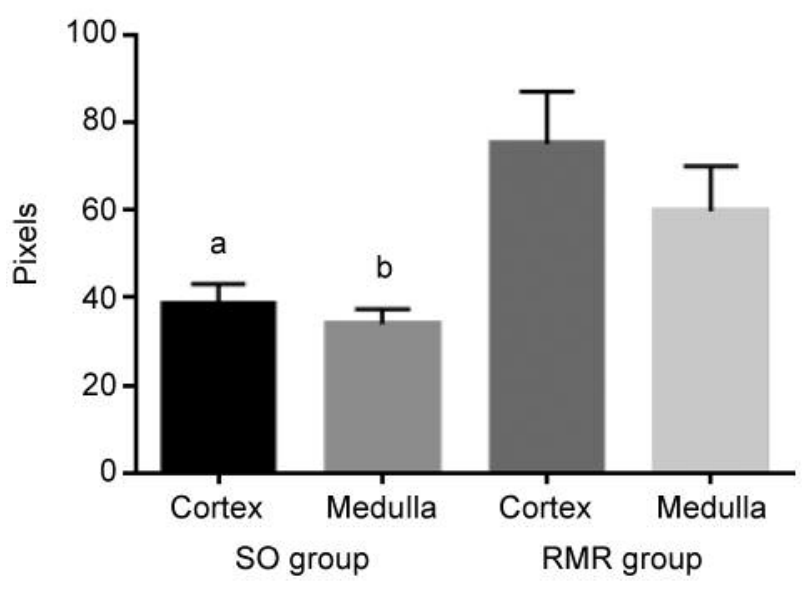

Figure 3. Evaluation of echogenicity in renal cortex and medulla in sham-operated (SO) and renal mass reduction (RMR) groups 6 months after the surgery. Significantly different at ${ }^{a} p<0.05$ versus cortex in the $R M R$ group, ${ }^{b} p<0.05$ versus medulla in RMR group. 
in vivo $30: 829-834(2016)$

Table I. Measurement of renal dimensions (mean \pm S.D.) by ultrasonography at 3 and 6 months after renal mass reduction (RMR).

Ultrasonographic measurement $(\mathrm{cm})$

\begin{tabular}{|c|c|c|c|c|c|c|c|c|}
\hline \multirow[t]{2}{*}{ Group } & \multicolumn{2}{|c|}{ Renal length } & \multicolumn{2}{|c|}{ Renal thickness } & \multicolumn{2}{|c|}{ Cortical thickness } & \multicolumn{2}{|c|}{ Medullary length } \\
\hline & 3 Months & 6 Months & 3 Months & 6 Months & 3 Months & 6 Months & 3 Months & 6 Months \\
\hline $\mathrm{SO}(\mathrm{n}=12)$ & $1.87 \pm 0.08^{a}$ & $1.96 \pm 0.16^{\mathrm{b}}$ & $1.15 \pm 0.07 \mathrm{a}$ & $1.18 \pm 0.11^{\mathrm{a}}$ & $0.39 \pm 0.04^{a}$ & $0.39 \pm 0.05^{\mathrm{a}}$ & $0.75 \pm 0.11^{\mathrm{a}}$ & $0.80 \pm 0.09^{a}$ \\
\hline $\operatorname{RMR}(\mathrm{n}=11)$ & $1.83 \pm 0.18^{\mathrm{a}}$ & $2.06 \pm 0.34^{b}$ & $1.44 \pm 0.16^{b}$ & $1.57 \pm 0.25^{b}$ & $0.48 \pm 0.12^{\mathrm{a}}$ & $0.58 \pm 0.17^{b}$ & $0.96 \pm 0.21^{b}$ & $0.99 \pm 0.18^{b}$ \\
\hline
\end{tabular}

SO: Sham-operated rats. For each parameter, different letters correspond to statistically significantly different values $(p<0.05)$.

Renal echogenicity. The cortical and medullary echogenicity was evaluated in US images from both experimental groups at 6 months of the experimental protocol. The cortical and medullary echogenicity was significantly higher in the RMR group when compared with the SO group $(p<0.05)$ (Figure $3)$.

Correlation of ultrasonographic parameters with the histological and biochemical parameters in RMR group. Only one statistically significant correlation was observed and was between the plasma creatinine and the renal length 3 months after RMR ( $\mathrm{r}=-0.612, p=0.045)$. However, moderate correlations were observed between: creatinine clearance and renal length $(\mathrm{r}=0.530, p=0.094)$; and proteinuria and renal thickness $(\mathrm{r}=0.451, p=0.164), 3$ months after RMR. Additionally, a moderate correlation was observed between glomerulosclerosis and cortical thickness ( $\mathrm{r}=0.461, p=0.131) 6$ months after RMR (Table III).

\section{Discussion}

The model of 5/6 nephrectomy in rats is a widely studied animal model of chronic renal failure that is close enough to the pathophysiological characteristics of human CKD (21). Hence, this model is used to study different aspects of CKD, namely to assess the potential renoprotective effects of new drugs $(8,9)$. Detection of renal damage and fibrosis in this model is very important in chronic studies and finding a noninvasive means of its detection would provide tremendous utility in experimental studies.

US is a real-time imaging modality commonly used in in vivo evaluation of the kidney, and is a relatively cheap, noninvasive and non-nephrotoxic modality (12). Therefore, we applied this imaging modality in conscious rats subjected to $5 / 6$ nephrectomy in order to follow-up the progression of renal damage in a chronic experimental study ( 6 months). To our knowledge, no studies have yet been performed in order to detect alterations in renal size and thickness and echogenicity of the renal cortex and medulla by US in this model.
Table II. Measurement of renal dimensions (mean \pm S.D.) in sham-operated $(\mathrm{SO})$ and renal mass reduction (RMR) groups by ultrasonography and anatomopathologically by ruler at the end of the experimental protocol.

\begin{tabular}{lccccc}
\hline Group & \multicolumn{2}{c}{ Renal length $(\mathrm{cm})$} & & \multicolumn{2}{c}{ Renal thickness $(\mathrm{cm})$} \\
\cline { 2 - 3 } \cline { 5 - 6 } & Ultrasound & Ruler & & Ultrasound & Ruler \\
\hline SO (n=12) & $1.96 \pm 0.16^{\mathrm{a}}$ & $1.91 \pm 0.24^{\mathrm{a}}$ & & $1.18 \pm 0.11^{\mathrm{a}}$ & $1.10 \pm 0.10^{\mathrm{b}}$ \\
RMR (n=11) & $2.06 \pm 0.34^{\mathrm{a}}$ & $1.52 \pm 0.23^{\mathrm{b}}$ & & $1.57 \pm 0.25^{\mathrm{c}}$ & $1.33 \pm 0.17^{\mathrm{d}}$ \\
\hline
\end{tabular}

For each parameter, different letters correspond to statistically significantly different values $(p<0.05)$.

Histopathological studies of renal tissue after renal mass reduction revealed the presence of three phases: I: fast hypertrophic phase ( 2 to 4 weeks after RMR); II: phase with minimal histological changes (4 to 10 weeks); and III: development of glomerular sclerosis and tubulointerstitial fibrosis (after 10 weeks) (22). Therefore, we started the evaluation of the kidneys by US 3 months after the 5/6 nephrectomy.

In general, the kidney dimensions (renal length, renal thickness, cortical thickness and medullary length) were higher in the RMR group when compared with the SO group at 3 and 6 months of the protocol. These results are in accordance with what would be expected, since compensatory kidney hypertrophy in the animals of the RMR group as a consequence of renal mass reduction was predictable (23). In both groups, the renal dimensions were higher at 6 months of the experimental protocol when compared with the same dimensions at 3 months of the protocol, which might be attributable to normal animal growth because renal size changes with age (24).

At the end of the experimental protocol, kidney dimensions were also measured using a ruler (anatomopathological dimensions). All dimensions of the kidney measured in this way were lower when compared to US dimensions. However, this may be due to the lack of blood supply that occurs immediately after the excision of the kidney from the animals, with a consequent reduction in 
Table III. Spearman correlation coefficients between ultrasonographic measurements and biochemical and histological parameters, at 3 months and at the end of the experimental protocol ( 6 months) in the renal mass reduction group ( $n=11)$.

\begin{tabular}{|c|c|c|c|c|c|c|c|c|c|c|c|c|}
\hline \multirow[t]{2}{*}{ Group } & \multicolumn{2}{|c|}{$\begin{array}{l}\text { Renal } \\
\text { length }\end{array}$} & \multicolumn{2}{|c|}{$\begin{array}{l}\text { Renal } \\
\text { thickness }\end{array}$} & \multicolumn{2}{|c|}{$\begin{array}{l}\text { Cortical } \\
\text { thickness }\end{array}$} & \multicolumn{2}{|c|}{$\begin{array}{l}\text { Medullary } \\
\text { length }\end{array}$} & \multicolumn{2}{|c|}{$\begin{array}{c}\text { Cortical } \\
\text { echogenicity }\end{array}$} & \multicolumn{2}{|c|}{$\begin{array}{l}\text { Medullary } \\
\text { echogenicity }\end{array}$} \\
\hline & 3 Months & 6 Months & 3 Months 6 & 6 Months & 3 Months & 6 Months & 3 Months & 6 Months 3 & 3 Months & 6 Months & 3 Months & 6 Months \\
\hline Proteinuria & 0.233 & 0.102 & 0.451 & 0.158 & -0.082 & 0.039 & -0.136 & 0.155 & nd & -0.357 & nd & 0.214 \\
\hline Plasma creatinine & $-0.612^{*}$ & -0.025 & -0.319 & -0.032 & -0.169 & 0.155 & -0.382 & -0.098 & nd & -0.071 & nd & -0.321 \\
\hline Creatinine clearance & 0.530 & 0.189 & 0.282 & -0.035 & 0.174 & -0.272 & 0.018 & 0.081 & nd & 0.393 & nd & 0.500 \\
\hline Glomerulosclerosis & nd & 0.031 & nd & 0.207 & nd & 0.461 & nd & 0.199 & nd & 0.418 & nd & -0.120 \\
\hline Interstitial fibrosis & nd & -0.140 & nd & 0.028 & nd & 0.056 & nd & -0.112 & nd & $\times$ & nd & $\times$ \\
\hline
\end{tabular}

*Statistically significant at $p<0.05 . \times$ : It was only possible to obtain three results for interstitial fibrosis hence it was not possible to determine the correlation coefficients; nd: not determined.

kidney dimensions. Abershon-butche et al. compared renal dimensions in horses obtained using the US method and post-mortem examination. These authors also observed that US technique overestimated the size of the kidneys (25).

The existing US equipment in our laboratory allows the clear differentiation of the cortex and medulla. However, it was not possible to determine cortical and medullary echogenicity at 3 months after RMR, primarily because of a problem with image quality at that time. Hence the cortical and medullary echogenicity was evaluated in both groups only at 6 months after RMR.

According to several researchers, variation of echogenicity may be an indicator of alteration in several tissues $(19,26-$ 28 ). In the kidney, an increase of echogenicity is considered an indicator of renal fibrosis (29). In the present study, the cortical and medullary echogenicity was significantly higher in the RMR group when compared with the SO group. These results seem to indicate that the echogenicity evaluated in either cortex or medulla is a good sensitive marker of fibrosis in the 5/6 nephrectomy model.

Previous work performed both in humans and animals with renal disease have determined the kidney dimensions by US in order to compare the data with histopathological findings (30-32). In this study we correlated US data with histopathological and also with biochemical findings. Although Moghazi et al. observed a significant correlation between echogenicity and histopathological data in humans (19), we did not observe a significant correlation between these two variables in Wistar rats. In this study, we observed only a statistically significant correlation between renal length and plasma creatinine 3 months after RMR. However, moderate correlations were observed between the creatinine clearance and the renal length; and proteinuria and the renal thickness, 3 months after RMR. Additionally, a moderate correlation was observed between the glomerulosclerosis and the cortical thickness 6 months after RMR. Contrarily to what was observed in humans $(19,31)$, the findings of this study seems to indicate that the US parameters are more associated with biochemical parameters of renal function than with histological alterations. However, an explanation for our results may be the low number of animals used in this study. Moreover, it would also have been important to evaluate other US parameters, such as resistive index, pulsatility index, peak systolic velocity and end-diastolic velocity, in order to better characterize the changes in kidney during CKD progression.

This preliminary study demonstrates that it is possible to image kidney in conscious rats by two-dimensional US and supports future application of this technique for monitoring the progression of renal damage in chronic studies with the RMR model, where anesthetic application is not possible. Unlike other procedures that can only be determined after the animals' sacrifice, the US evaluation of the kidney may be performed more than once, at any time during the study.

\section{Conflicts of Interest}

Nothing to disclose.

\section{Acknowledgements}

This work was supported by : European Investment Funds by FEDER/COMPETE/POCI Operational Competitiveness and Internationalization Programme, under Project POCI-01-0145-FEDER-006958 and National Funds by FCT - the Portuguese Foundation for Science and Technology, under project UID/AGR/04033/2013; and by European Investment Funds by FEDER/COMPETE/POCI Operational Competitiveness and Internationalization Programme, under Project POCI-01-0145-FEDER-016728 and National Funds by FCT - the Portuguese Foundation for Science and Technology, under the project PTDC/DTP-DES/6077/2014.

\section{References}

1 Jha V, Garcia-Garcia G, Iseki K, Li Z, Naicker S, Plattner B, Saran R, Wang AYM and Yang CW: Chronic kidney disease: global dimension and perspectives. Lancet 382: 260-272, 2013. 
2 Yang H-C, Zuo Y and Fogo A: Models of chronic kidney disease. Drug Discov Today Dis Models 7: 13-19, 2010.

3 Ortiz A, Sanchez-Niño MD, Izquierdo MC, Martin-Cleary C, Garcia-Bermejo L, Moreno JA, Ruiz-Ortega M, Draibe J, Cruzado JM, Garcia-Gonzalez MA, Lopez-Novoa JM, Soler MJ and Sanz AB: Translational value of animal models of kidney failure. Eur J Pharmacol 759: 205-220, 2015.

4 Anderson S, Meyer TW, Rennke HG and Brenner BM: Control of glomerular hypertension limits glomerular injury in rats with reduced renal mass. J Clin Invest 76: 612-619, 1985.

5 Bidani AK, Schwartz MM and Lewis EJ: Renal autoregulation and vulnerabitity to hypertensive injury in remmant kidney. Am J Physiol 252: F1003-F1010, 1987.

6 Fogo A and Ichikawa I: Evidence for the central role of glomerular growth in the development of sclerosis. Semin Nephrol 9: 329-342, 1989.

7 Tapia E, Gabbai FB, Calleja C, Franco M, Cermeño JL, Bodadilla NA, Pérez JM, Alvarado JÁ and Herrera-Costa J: Determinants of renal damage in rats with systemic hypertension and partial renal ablation. Kidney Int 38: 642-648, 1990.

8 Pires MJ, Rodríguez-Peña AB, Miguel A, Cenador B, Evangelista S, Esteller A, Sánchez-Rodríguez A, Colaço A and López-Novoa JM: Long-term nebivolol administration reduces renal fibrosis and prevents endothelial dysfunction in rats with hypertension induced by renal mass reduction. J Hypertens 25 : 2486-2496, 2007.

9 Cho K-H, Kim H-J, Rodriguez-Iturbe B and Vaziri ND: Niacin ameliorates oxidative stress, inflammation, proteinuria, and hypertension in rats with chronic renal failure. Am J Physiol Renal Physiol 297: F106-F113, 2009.

10 Farris AB and Colvin RB: Renal interstitial fibrosis: mechanisms and evaluation. Curr Opin Nephrol Hypertens 21: 289-300, 2012.

11 Farris AB and Alpers CE: What is the best way to measure renal fibrosis?: A pathologist's perspective. Kidney Int Suppl 4: 9-15, 2014.

12 Kolofousi C, Stefanidis K, Cokkinos DD, Karakitsos D, Antypa E and Piperopoulos P: Ultrasonographic Features of Kidney Transplants and Their Complications: An Imaging Review. ISRN Radiol DOI http://dx.doi.org/10.5402/2013/480862, 2012.

13 Grassi W, Filippucci E and Busilacchi P: Musculoskeletal ultrasound. Best Pract Res Clin Rheumatol 18: 813-826, 2004.

14 Sanusi AA, Arogundade FA, Famurewa OC, Akintomide AO, Soyinka FO, Ojo OE and Akinsola A: Relationship of ultrasonographically determined kidney volume with measured GFR, calculated creatinine clearance and other parameters in chronic kidney disease (CKD). Nephrolol Dial Transplantat 24: 1690-1694, 2009.

15 Wang YXJ, Betton G, Floettmann E, Fantham E and Ridgwell G: Imaging kidney in conscious rats with high-frequency ultrasound and detection of two cases of unilateral congenital hydronephrosis. Ultrasound Med Biol 33: 483-486, 2007.

16 Ablett MJ, Coulthard A, Lee RE, Richardson DL, Bellas T, Owen JP, Keir MJ and Butler TJ: How reliable are ultrasound measurements of renal length in adults? Br J Radiol 68: 10871089, 1995.

17 Emamian SA, Nielsen MB and Pedersen JF: Intraobserver and interobserver variations in sonographic measurements of kidney size in adult volunteers. A comparison of linear measurements and volumetric estimates. Acta Radiol 36: 399-401, 1995.
18 d'Anjou MA: Kidneys and Ureters. In: Atlas of small Animal Ultrasonography. Pennink D and d'Anjou MA (eds.). Iowa, Blackwell Publishing, pp. 339-364, 2008.

19 Moghazi S, Jones E, Schroepple J, Arya K, McClellan W, Hennigar RA and O'Neill WC: Correlation of renal histopathology with sonographic findings. Kidney Int 67: 1515$1520,2005$.

20 Asaba K, Tojo A, Onozato ML, Kinugasa S, Miyazaki H, Miyashita K, Uehara Y, Hirata Y, Kimura K, Goto A, Omata M and Fujita T: Long-term renal prognosis of $\operatorname{IgA}$ nephropathy with therapeutic trend shifts. Intern Med 48: 883-890, 2009.

21 Tapia E, Franco M, Sánchez-Lozada LG, Soto V, Avila-Casado C, Santamaría J, Quiroz Y, Rodríguez-Iturbe B and HerreraAcosta J: Mycophenolate mofetil prevents arteriolopathy and renal injury in subtotal ablation despite persistent hypertension, Kidney Int 63: 994-1002, 2003.

22 Waldherr R and Gretz N: Natural course of the development of histological lesions after 5/6 nephrectomy. Contrib Nephrol 60: 64-72, 1988.

23 Fleck C, Appenroth D, Jonas P, Koch M, Kundt G, Nizze H and Stein G: Suitability of $5 / 6$ nephrectomy $(5 / 6 \mathrm{NX})$ for the induction of interstitial renal fibrosis in rats - Influence of sex, strain, and surgical procedure. Exp Toxicol Pathol 57: 195-205, 2006.

24 Celsi G, Jakobsson B and Aperia A: Influence of age on compensatory renal growth in rats. Pediatr Res 20:347-350, 1986.

25 Habershon-Butcher J, Bowen M and Hallowell G: Validation of a novel translumbar ultrasound technique for measuring renal dimensions in horses. Vet Radiol Ultrasound 55: 323-330, 2014.

26 Rosenfield A and Siegel N: Renal parenchymal disease: histopathologic-sonographic correlation. AJR Am J Roentgenol 137: 793-798, 1981.

27 Faustino-Rocha AI, Oliveira PA, Duarte JA, Ferreira R and Ginja M: Ultrasonographic evaluation of gastrocnemius muscle in a rat model of N-methyl-N-nitrosourea-induced mammary tumor. In Vivo 27: 803-807, 2013.

28 Hricak H, Cruz C, Romanski R, Uniewski MH, Levin NW, Madrazo BL, Sandler MA and Eyler WR: Renal parenchymal disease - sonographic-histologic correlation. Radiology 144: 141-147, 1982.

29 Buturovic-Ponikvar J and Visnar-Perovic A: Ultrasonography in chronic renal failure. Eur J Radiol 46: 115-122, 2003.

30 Beland MD, Walle NL, Machan JT and Cronan JJ: Renal cortical thickness measured at ultrasound: Is it better than renal length as an indicator of renal function in chronic kidney disease? AJR Am J Roentgenol 195: W146-W149, 2010.

31 Chen QK, He F, Feng XR, Luo ZM, Zhang JL, Zhang L, Wang $\mathrm{Y}$ and Tong JR: Correlation of Doppler parameters with renal pathology: A study of 992 patients. Exp Ther Med 7: 439-442, 2014.

32 Onyeanusi B, Adeniyi A, Onyeanusi C, Ayo J and Ibe C: A study of the kidney of the Wistar rat in Northern Guinea Savannah zone: the morphometric aspect. Pakistan J Nut 8: 1040-1042, 2009.

Received September 26, 2016

Revised October 9, 2016

Accepted October 10, 2016 